\title{
One Particle Transitions and Correlation in Quantum Mechanics
}

\author{
A. R. Ruffa \\ (October 9, 1964)
}

\begin{abstract}
The quantum mechanical correlation problem, involving the evaluation of expectation values of many particle operators for a given eigenstate, is formulated, by means of the matrix sum rule, in terms of unobservable or virtual one particle transitions to other eigenstates of the system. This formulation is set forth for the two-fold purpose of both demonstrating the nature of the many particle correlations from the point of view of matrix mechanics, and making possible the derivation of sum rules which may be used in their analysis. The analysis shows that the transition integrals $\left\langle\alpha\left|\mathbf{A}_{i}\right| \alpha^{\prime}\right\rangle$ and $\left\langle\alpha\left|\mathbf{A}_{j}\right| \alpha^{\prime}\right\rangle$, where $\mathbf{A}_{i}$ and $\mathbf{A}_{j}$ are arbitrary one particle operators involving the $i$ th and $j$ th particles, respectively, are not necessarily equal, since such transitions can involve quantum states of different coordinate symmetry, i.e., the antisymmetric and symmetric states when $N=2$, and the states of mixed symmetry, in addition, when $N \geq 3$. The indistinguishability principle is demonstrated to require only that
\end{abstract}

$$
\sum_{\alpha^{\prime}}\left|\left\langle\alpha\left|\mathbf{A}_{i}\right| \alpha^{\prime}\right\rangle\right|^{2}=\sum_{\alpha^{\prime}}\left|\left\langle\alpha\left|\mathbf{A}_{j}\right| \alpha^{\prime}\right\rangle\right|^{2} .
$$

By detailed analysis of one particle transitions to states of various symmetries, the relationship between the various one particle transitions is determined. The expectation value for an antisymmetric state of the two particle operator $\sum_{i \neq j} \mathbf{A}_{i} \cdot \mathbf{A}_{i}$ is found to be

$$
\sum_{i \neq j}\left\langle\alpha\left|\mathbf{A}_{i} \cdot \mathbf{A}_{j}\right| \alpha\right\rangle=N(N-1) \sum_{\alpha_{A}^{\prime}}\left|\left\langle\alpha\left|\mathbf{A}_{1}\right| \alpha_{A}^{\prime}\right\rangle\right|^{2}-N \sum_{\alpha_{N}^{\prime}}\left|\left\langle\alpha_{A}\left|\mathbf{A}_{1}\right| \alpha_{N}^{\prime}\right\rangle\right|^{2}
$$

where the sum $\alpha_{A}^{\prime}$ is over all antisymmetric states, and the sum $\alpha_{N}^{\prime}$ is over all the nonantisymmetric states. This equation is used to obtain an approximate relation between these expectation values and observable quantities.

From the Heisenberg commutation relations, the sum rule

$$
\sum_{i=1}^{N} \sum_{\alpha^{\prime}}\left(E_{\alpha^{\prime}}-E_{\alpha}\right)|<\alpha| \mathbf{r}_{i}\left|\alpha^{\prime}>\right|^{2}=3 \AA^{2} N / 2 m
$$

is obtained and used to demonstrate some general properties of systems in the nonrelativistic approximation.

\section{Introduction}

Transitions in quantum mechanics usually refer to the change of an $N$ particle system from one quantum state to another. Such a transition involving all the particles of the system is observable by radiative processes in principle if it is not prohibited by some selection rule. On the other hand, transitions not involving all the particles of the system have received little attention because they do not involve an observable property of the system. In the realm of scientific analysis, however, there are many occasions when it is desirable, if not expedient, to study a system in terms of properties which are not observable, but rather, are a product of the imagination - a method of inquiry analogous to a study of the rhinoceros by way of the properties of the unicorn and the dragon.

In line with this, the value of a discussion of such transitions lies in their connection with an unobservable property of the system, which nevertheless, because of its importance, has been the object of considerable interest. The property of interest concerns the effects resulting from the correlations of the particles of the system. The interest in these unobservable effects has arisen from the success of the relatively simple independent particle approximation in the description of the properties of a many particle system. While the independent particle model has proven to be an excellent first approximation, the limitations of this approach lie 
mainly in its inability to properly evaluate the correlation effects. Consequently, the correlation problem concerns the attempt to arrive at a more accurate description of many particle systems.

From a mathematical point of view, the quantum mechanical correlation problem is associated with the evaluation of the expectation value, for a given quantum state, of any arbitrary many (two or more) particle operator - an expectation value which cannot be properly evaluated in the independent particle approximation. In the formalism of matrix mechanics, such an expectation value takes the form of a sum of products of matrix elements for the virtual one particle transitions. Consequently, in this article, the discussion of the one particle transitions is primarily intended to illustrate the nature of the many particle correlations from the matrix expansion point of view. In part 2, a detailed discussion of the requirements of the indistinguishability principle concerning the one particle transitions is included to elucidate some of their properties. Part 3 will demonstrate the detailed relationship between one particle transitions, while part 4 will give a discussion of the nature of two particle correlations in terms of the one particle transitions. Finally, in part 5, a sum rule which involves one particle transitions is obtained and used to demonstrate some general properties of systems in the nonrelativistic approximation. It is hoped that this somewhat different analysis of the correlation problem may introduce a fresh approach from which new ideas may evolve regarding this important topic.

\section{Requirements of the Indistinguishability Principle in the Case of One Particle Transitions}

If one considers the operator $\mathbf{A}=\sum_{i=1}^{N} \mathbf{A}_{i}$ for an $N$ particle system where $\mathbf{A}_{i}$ operates on the ith particle, the principle of the indistinguishability of like particles requires that the expectation value

$$
\left\langle\alpha\left|\mathbf{A}_{i}\right| \alpha\right\rangle=\int \psi_{\alpha}^{*}\left(\mathbf{x}_{1} \mathbf{x}_{2} \ldots \mathbf{x}_{N}\right) \mathbf{A}_{i} \psi_{\alpha}\left(\mathbf{x}_{1} \mathbf{x}_{2} \ldots \mathbf{x}_{N}\right) d \mathbf{x}_{1} \ldots d \mathbf{x}_{N}
$$

be equal to $\left\langle\alpha\left|\mathbf{A}_{j}\right| \alpha\right\rangle$ where $i \neq j$. Here $\psi_{\alpha}\left(\mathbf{x}_{1} \mathbf{x}_{2} \ldots \mathbf{x}_{N}\right)$ is the $N$ particle wave function describing the quantum state $\alpha$ of the $N$ particle system. In the following discussion, this state will be assumed to refer to an electronic system, since this assumption involves no loss of generality. Therefore, as a consequence of the indistinguishability principle, the expectation value of $\mathbf{A}$ for a given state $\psi_{\alpha}$ is given by

$$
\langle\alpha|\mathbf{A}| \alpha\rangle=N\left\langle\alpha\left|\mathbf{A}_{1}\right| \alpha\right\rangle \text {. }
$$

The question may now be raised as to the relationship between $\left\langle\alpha\left|\mathbf{A}_{i}\right| \alpha^{\prime}\right\rangle$ and $\left\langle\alpha\left|\mathbf{A}_{j}\right| \alpha^{\prime}\right\rangle$ where the state $\psi_{\alpha^{\prime}}$ differs from $\psi_{\alpha}$. If, in analogy with the above discussion, one succumbs to the temptation of concluding that $\left\langle\alpha\left|\mathbf{A}_{i}\right| \alpha^{\prime}\right\rangle=\left\langle\alpha\left|\mathbf{A}_{j}\right| \alpha^{\prime}\right\rangle$, then one is immediately confronted with a contradiction in the evaluation of the expectation value of a many particle operator for a given quantum state.

This may be seen in the following manner. According to the sum rule for matrix multiplication, the expectation value for the two particle operator $\mathbf{A}_{i} \cdot \mathbf{A}_{j}$ evaluated for the state $\psi_{\alpha}$ is given by

$$
\left\langle\alpha\left|\mathbf{A}_{i} \cdot \mathbf{A}_{j}\right| \alpha\right\rangle=\sum_{\alpha^{\prime}}\left\langle\alpha\left|\mathbf{A}_{i}\right| \alpha^{\prime}\right\rangle\left\langle\alpha^{\prime}\left|\mathbf{A}_{j}\right| \alpha\right\rangle
$$

where the sum is over all the eigenstates of the Hamiltonian. In the case when $i=j$, we are led to the relation

$$
\left\langle\alpha\left|\mathbf{A}_{i}^{2}\right| \alpha\right\rangle=\sum_{\alpha^{\prime}}\left|\left\langle\alpha\left|\mathbf{A}_{i}\right| \alpha^{\prime}\right\rangle\right|^{2}
$$


since $\mathbf{A}_{i}$ is assumed to be Hermitian. If the relation $\left\langle\left|\mathbf{A}_{i}\right| \alpha^{\prime}\right\rangle=\left\langle\alpha\left|\mathbf{A}_{j}\right| \alpha^{\prime}\right\rangle$ is always correct, then comparison of (2) and (3) leads to the conclusion that $\left\langle\alpha\left|\mathbf{A}_{i}^{2}\right| \alpha\right\rangle=\left\langle\alpha\left|\mathbf{A}_{i} \cdot \mathbf{A}_{j}\right| \alpha\right\rangle$, which is not true in general. For example, the quantity $\left\langle\alpha\left|\mathbf{r}_{i}^{2}\right| \alpha\right\rangle$ for the ground state of a free atom is proportional to its diamagnetic susceptibility, and is usually an order of magnitude greater than $\left\langle\alpha\left|\mathbf{r}_{i} \cdot \mathbf{r}_{j}\right| \alpha\right\rangle$, a small quantity which vanishes for a free atom in the independent particle approximation. Similarly, the small quantity $\left\langle\alpha\left|\mathbf{p}_{i} \cdot \mathbf{p}_{j}\right| \alpha\right\rangle$ vanishes for any bound state in the independent particle approximation, while $\left\langle\alpha\left|\mathbf{p}_{i}^{2}\right| \alpha\right\rangle$, by the quantum mechanical virial theorem, is proportional to the total energy of the state $\psi_{\alpha}$ for any system described by a Hamiltonian which includes only Coulombic interactions. Consequently, the equality of the one particle transitions $\left\langle\alpha\left|\mathbf{A}_{i}\right| \alpha^{\prime}\right\rangle$ and $\left\langle\alpha\left|\mathbf{A}_{j}\right| \alpha^{\prime}\right\rangle$ would rule out the applicability of the matrix sum rule when applied to the determination of the expectation value of any two or more particle operator.

It is important to note that the indistinguishability principle, itself, does not require that $\left\langle\alpha\left|\mathbf{A}_{i}\right| \alpha^{\prime}\right\rangle$ equal $\left\langle\alpha\left|\mathbf{A}_{j}\right| \alpha^{\prime}\right\rangle$. This is easily demonstrated by using the sum rule for matrix multiplication which indicates that

$$
\left\langle\alpha\left|\mathbf{A}_{i}^{2}\right| \alpha\right\rangle=\sum_{\alpha^{\prime}}\left|\left\langle\alpha\left|\mathbf{A}_{i}\right| \alpha^{\prime}\right\rangle\right|^{2}
$$

and

$$
\left\langle\alpha_{j}\left|\mathbf{A}_{j}^{2}\right| \alpha\right\rangle=\sum_{\alpha^{\prime}}\left|\left\langle\alpha\left|\mathbf{A}_{j}\right| \alpha^{\prime}\right\rangle\right|^{2} .
$$

According to the indistinguishability principle, the left hand sides of (3) and (4) are equal. Consequently, the indistinguishability principle requires only that

$$
\sum_{\alpha^{\prime}}|<\alpha| \mathbf{A}_{j}\left|\alpha^{\prime}>\right|^{2}=\sum_{\alpha^{\prime}}\left|\left\langle\alpha\left|\mathbf{A}_{i}\right| \alpha^{\prime}\right\rangle\right|^{2} .
$$

\section{Virtual One Particle Transitions Between States of Different Particle Coordinate Symmetry}

The investigation into a more detailed relation between the $\left\langle\alpha\left|\mathbf{A}_{i}\right| \alpha^{\prime}\right\rangle$ and the $\left\langle\alpha\left|\mathbf{A}_{j}\right| \alpha^{\prime}\right\rangle$ than can be obtained from the indistinguishability principle leads immediately into a consideration of one particle transitions between states of different coordinate symmetry. As has been discussed before in some detail, ${ }^{1}$ the eigenfunctions of an $N$ particle Hamiltonian fall into three or more noncombining sets with different symmetry properties. The eigenfunctions of one set are antisymmetric with respect to particle interchanges, so that $\mathbf{P}_{i j} \psi_{A}=-\psi_{A}$, where $\mathbf{P}_{i j}$ is the permutation operator which interchanges the $i$ th and $j$ th electrons. Another set of eigenfunctions is symmetric, so that $\mathbf{P}_{i j} \psi_{i j}=\psi_{S}$. In addition, when $\mathbf{N} \geq 3$, there exist other mixed symmetry types $\psi_{M}$ which are neither symmetric nor antisymmetric. It is evident that while the $\psi_{A}$ and the $\psi_{S}$ are eigenfunctions of all the $\mathbf{P}_{i j}$, the $\psi_{M}$ are not since $\mathbf{P}_{i j}^{2} \psi=\psi$, and therefore if $\mathbf{P}_{i j} \psi=c \psi$, then $c= \pm 1$. However, mixed functions which are eigenfunctions of the $\mathbf{P}_{i j}$ individually can be constructed, since it is evident that $\psi_{M}+\mathbf{P}_{i j} \psi_{M}$ and $\psi_{M}-\mathbf{P}_{i j} \psi_{M}$ are symmetric and antisymmetric in the interchange of particles $i$ and $j$, respectively. This property results from the fact that each $\mathbf{P}_{i j}$ commutes with the Hamiltonian, so that it is always possible to construct their simultaneous eigenfunctions.

In a real transition, i.e., one which is observable, at least in principle, in which the operator involved $\mathbf{A}=\sum_{i=1}^{N} \mathbf{A}_{i}$ is symmetric in all the particles, the matrix elements between states of different symmetry properties vanish. For an electronic system, it is well known that the wave functions belong to the antisymmetric set, so that real transitions can only occur between antisymmetric states. However, in a virtual one particle transition, this restriction

1 E. Wigner, Z. Physik 40, 492 (1927); 40, 883 (1927); 43, 624 (1927); F. Hund, Z. Physik 43, 778 (1927); W. Heisenberg, Z. Physik 41, 239 (1927); E. E. Witmer and J. P. Vinti, Phys. Rev. 47, 538 (1935). 
does not exist. In fact, as was seen above, a sum over all possible one particle transitions, i.e., those which involve transitions to all the eigenstates of the Hamiltonian, can only be meaningful if it includes transitions between states of different symmetry. This means that for an electronic system, one must consider, in addition to transitions to antisymmetric states, for which $\left\langle\alpha_{A}\left|\mathbf{A}_{i}\right| \alpha_{A}^{\prime}\right\rangle$ and $\left\langle\alpha_{A}\left|\mathbf{A}_{j}\right| \alpha_{A}^{\prime}\right\rangle$ are equal, transitions of the type $\left\langle\alpha_{A}\left|\mathbf{A}_{i}\right| \alpha_{S}^{\prime}\right\rangle$ and, for the case when $N \geq 3$, transitions of the type $\left\langle\alpha_{A}\left|\mathbf{A}_{i}\right| \alpha_{M}^{\prime}\right\rangle$ as well. ${ }^{2}$ It is apparent from this discussion that the expectation value for an antisymmetric state of a two particle operator cannot be completely defined in terms of one particle transitions between antisymmetric eigenstates of the Hamiltonian alone.

It is now necessary to analyze in detail the relationship between one particle transitions involving states of different symmetry. Since the cases $N=2$ and $N \geq 3$ are somewhat different, they will be studied separately.

\subsection{One Particle Transitions for Two Electron Systems}

In the case of a two particle system, the eigenfunctions of the Hamiltonian are either symmetric or antisymmetric. We assume a two electron system to be in the antisymmetric state $\psi_{\alpha}$. If one considers the transition element $\left\langle\alpha\left|\mathbf{A}_{1}\right| \alpha^{\prime}\right\rangle$ and then applies the permutation operator $P_{12}$ to the integral, one obtains $\left\langle-\alpha\left|\mathbf{A}_{2}\right|-\alpha^{\prime}\right\rangle=\left\langle\alpha\left|\mathbf{A}_{2}\right| \alpha^{\prime}\right\rangle$ if the state $\psi_{\alpha^{\prime}}$ is also antisymmetric. However, one obtains $\left\langle-\alpha\left|\mathbf{A}_{2}\right| \alpha^{\prime}\right\rangle=-\left\langle\alpha\left|\mathbf{A}_{2}\right| \alpha^{\prime}\right\rangle$ if the state $\psi_{\alpha^{\prime}}$ is symmetric. Since the integral must be invariant under interchanges of the dummy indices 1 and 2 , we conclude for a two electron system that

and

$$
\left\langle\alpha_{A}\left|\mathbf{A}_{1}\right| \alpha_{A}^{\prime}\right\rangle=\left\langle\alpha_{A}\left|\mathbf{A}_{2}\right| \alpha_{A}^{\prime}\right\rangle
$$

$$
\left\langle\alpha_{A}\left|\mathbf{A}_{1}\right| \alpha_{S}^{\prime}\right\rangle=-\left\langle\alpha_{A}\left|\mathbf{A}_{2}\right| \alpha_{S}^{\prime}\right\rangle
$$

for one electron transitions between two antisymmetric states and between an antisymmetric state and a symmetric state, respectively. ${ }^{3}$

\subsection{One Particle Transitions for Systems of Three or more Electrons}

For systems of three or more electrons, an analysis similar to that made above indicates that for one particle transitions between antisymmetric states only, $\left\langle\alpha_{A}\left|\mathbf{A}_{i}\right| \alpha_{A}^{\prime}\right\rangle=\left\langle\alpha_{A}\left|\mathbf{A}_{j}\right| \alpha_{A}^{\prime}.\right\rangle$, while the one particle transition integrals between symmetric and antisymmetric states vanish. The validity of the latter statement may be seen by considering transitions between symmetric and antisymmetric states involving the operators $\mathbf{A}_{i}, \mathbf{A}_{j}$, and $\mathbf{A}_{k}$, where $i, j$, and $k$ are different indices. We note that ${ }^{3 a}$

$$
\begin{aligned}
& \mathbf{P}_{i j}\left\langle\alpha_{A}\left|\mathbf{A}_{i}\right| \alpha_{S}^{\prime}\right\rangle=\left\langle\alpha_{A}\left|\mathbf{A}_{i}\right| \alpha_{S}^{\prime}\right\rangle=-\left\langle\alpha_{A}\left|\mathbf{A}_{j}\right| \alpha_{S}^{\prime}\right\rangle \\
& \mathbf{P}_{i k}\left\langle\alpha_{A}\left|\mathbf{A}_{i}\right| \alpha_{S}^{\prime}\right\rangle=\left\langle\alpha_{A}\left|\mathbf{A}_{i}\right| \alpha_{S}^{\prime}\right\rangle=-\left\langle\alpha_{A}\left|\mathbf{A}_{k}\right| \alpha_{S}^{\prime}\right\rangle \\
& \mathbf{P}_{j k}\left\langle\alpha_{A}\left|\mathbf{A}_{j}\right| \alpha_{S}^{\prime}\right\rangle=\left\langle\alpha_{A}\left|\mathbf{A}_{j}\right| \alpha_{S}^{\prime}\right\rangle=-\left\langle\alpha_{A}\left|\mathbf{A}_{k}\right| \alpha_{S}^{\prime}\right\rangle .
\end{aligned}
$$

The only possible way for (8), (9), and (10) to be simultaneously correct is if

$$
\left\langle\alpha_{A}\left|\mathbf{A}_{i}\right| \alpha_{S}^{\prime}\right\rangle=\left\langle\alpha_{A}\left|\mathbf{A}_{j}\right| \alpha_{S}^{\prime}\right\rangle=\left\langle\alpha_{A}\left|\mathbf{A}_{k i}\right| \alpha_{S}^{\prime}\right\rangle=0 .
$$

\footnotetext{
${ }^{2}$ For a system of Bosons, which are described by symmetric wave functions, one must consider in addition to the $\left\langle\alpha_{s}\left|\mathbf{A}_{i}\right| \alpha_{S}^{\prime}\right\rangle$, transitions of the type $>\alpha_{s}\left|\mathbf{A}_{i}\right| \alpha_{A}^{\prime}>$ and $\left\langle\alpha_{s}\left|\mathbf{A}_{i}\right| \alpha_{M}^{\prime}>\right.$.

${ }^{3}$ If one were discussing a system of two Bosons, the above rules would hold in reverse order, i.e., one particle transition integral between two symmetric states would be equal, while one particle transition integral between a symmetric and an antisymmetric state would have opposite signs.

3a The operation $\mathbf{P}_{i j}\left\langle\alpha\left|A_{i}\right| \alpha^{\prime}\right\rangle$ is equivlent to the transformation $\left\langle\alpha\left|\mathbf{P}_{i j^{2}} A_{i} \mathbf{P}_{i j^{2}}\right| \alpha^{\prime}\right\rangle$ since it is evident that $\left\langle\alpha\left|\mathbf{P}_{i j}^{-1} A_{i} \mathbf{P}_{i j}\right| \alpha^{\prime}\right\rangle=$ $\left\langle\alpha\left|\mathbf{P}_{i j} A_{i} \mathbf{P}_{i j}\right| \alpha^{\prime}\right\rangle=\left\langle\alpha\left|A_{j}\right| \alpha^{\prime}\right\rangle$.
} 
By allowing the indices $i, j$, and $k$ to take on all possible combinations of values from 1 to $N$, one may see that all the one electron transitions between an antisymmetric and a symmetric state vanish.

There now remains for analysis the case of the virtual one partlcle transitions between the antisymmetric and the mixed states $\psi_{M}$. Since, as was seen above, there are no one particle transitions between antisymmetric and symmetric states when $N \geq 3$, the transitions between

the antisymmetric and mixed states are decisive in providing a meaningiul application of the sum rule for matrix multiplication as used in eqs (2), (3), and (4). However, because the mixed states are not eigenfunctions of the permutation operator, there is no simple relationship connecting the transition elements involving the operators $\mathbf{A}_{i}$ and $\mathbf{A}_{j}$. This may be demonstrated by noting that since the permutation and Hamiltonian operators commute, then

$$
\mathbf{P}_{i j}\left(\mathbf{H} \psi_{M \alpha}\right)=\mathbf{H}\left(\mathbf{P}_{i j} \psi_{M \alpha}\right)=E_{\alpha}\left(\mathbf{P}_{i j} \psi_{M \alpha}\right) .
$$

Consequently, $\mathbf{P}_{i j} \psi_{M \alpha}$ is also an eigenfunction of the Hamiltonian having the same energy as $\psi_{M \alpha}$, so that $\mathbf{P}_{i j} \psi_{M \alpha}$ must be some linear combination of the degenerate eigenfunctions of the energy level $E_{\alpha}$. Because of this equivalence degeneracy, which cannot be removed by any perturbation symmetric in all the electrons, the relationship between transition integrals involving the operators $\mathbf{A}_{i}$ and $\mathbf{A}_{j}$ is not a simple proportionality, but instead, involves linear combinations of transition integrals to the various degenerate states.

It is, however, possible to derive a relationship between these transition integrals which is more detailed than that given by the indistinguishability principle in eq (5). That the interchange of two particles in a $\psi_{M \alpha}$ yields a linear combination of mixed wave functions which are degenerate with $\psi_{M \alpha}$ may be expressed in the following way:

$$
\mathbf{P}_{\imath j} \psi_{M \alpha l}=\sum_{l^{\prime}} t_{l l^{\prime}} \psi_{M \alpha l^{\prime}}
$$

where the sum is over all the states of the $l$-fold degenerate level $E_{\alpha}$. Equation (12) in matrix notation is

$$
\mathbf{P}_{i j}\left(\psi_{M \alpha l}\right)=\left(\psi_{M \alpha l}\right) \mathbf{T}
$$

where $\left(\psi_{M \alpha l}\right)$ is the $1 \times l$ row vector composed of the linearly independent $\psi_{M \alpha l}$. That the $l \times l$ matrix $\mathbf{T}$ is nonsingular, and is, in fact, its own inverse is easily seen since

or

$$
\mathbf{P}_{i j}^{2}\left(\psi_{M \alpha l}\right)=\left(\psi_{M \alpha l}\right)=\left(\psi_{M \alpha l}\right) \mathbf{T T}
$$

$$
\mathbf{T T}=1 .
$$

Moreover, because $\mathbf{T}^{2}$ is real, $\mathbf{T}$ must also be real.

We now construct the $1 \times l$ row matrix composed on the one particle transitions between the antisymmetric state $\psi_{A \alpha}$ and the mixed states of the $l$-fold degenerate energy level $E_{\alpha^{\prime}}$, i.e., $\left.\left(\left\langle\alpha_{A}\left|\mathbf{A}_{i}\right| \alpha_{M_{1}}^{\prime}\right\rangle \ldots<\alpha_{A}\left|\mathbf{A}_{i}\right| \alpha_{M_{l}}^{\prime}\right\rangle\right)$. From (13), it is evident that

$$
\begin{aligned}
& \mathbf{P}_{i j}\left(\left\langle\alpha_{A}\left|\mathbf{A}_{i}\right| \alpha_{M_{1}}^{\prime}>\ldots<\alpha_{A}\left|\mathbf{A}_{i}\right| \alpha_{M_{l}}^{\prime}\right\rangle\right) \\
& =\left(\left\langle\alpha_{A}\left|\mathbf{A}_{i}\right| \alpha_{M_{1}}^{\prime}>\ldots<\alpha_{A}\left|\mathbf{A}_{i}\right| \alpha_{M_{l}}^{\prime}\right\rangle\right) \\
& \left.=\left(\left\langle\alpha_{A}\left|\mathbf{A}_{j}\right| \alpha_{M_{1}}^{\prime}\right\rangle \ldots<\alpha_{A}\left|\mathbf{A}_{j}\right| \alpha_{M_{l}}^{\prime}\right\rangle\right) \mathbf{T} .
\end{aligned}
$$

The complex conjugate of the matrix $\left(\left\langle\alpha_{A}\left|\mathbf{A}_{i}\right| \alpha_{M_{1}}^{\prime}\right\rangle \ldots\left\langle\alpha_{A}\left|\mathbf{A}_{i}\right| \alpha_{M_{l}}^{\prime}\right\rangle\right)$ is the $l \times 1$ column matrix

$$
\left(\begin{array}{c}
\left\langle\alpha_{M_{1}}^{\prime}\left|\mathbf{A}_{i}\right| \alpha_{A}>\right. \\
\vdots \\
\left\langle\alpha_{M_{l}}^{\prime}\left|\dot{\mathbf{A}}_{i}\right| \alpha_{A}\right\rangle
\end{array}\right)
$$


From (16), one may see that

$$
\mathbf{P}_{i j}\left(\begin{array}{c}
\left\langle\alpha_{M_{1}}^{\prime}\left|\mathbf{A}_{i}\right| \alpha_{A}\right\rangle \\
\vdots \\
\left\langle\alpha_{M_{l}}^{\prime}\left|\mathbf{A}_{i}\right| \alpha_{A}\right\rangle
\end{array}\right)=\left(\begin{array}{c}
\left.<\alpha_{M_{1}}^{\prime}\left|\mathbf{A}_{i}\right| \alpha_{A}\right\rangle \\
\vdots \\
\left.<\alpha_{M_{l}}^{\prime}\left|\mathbf{A}_{i}\right| \alpha_{A}\right\rangle
\end{array}\right)=-\mathbf{T}\left(\begin{array}{c}
\left.<\alpha_{M_{1}}^{\prime}\left|\mathbf{A}_{i}\right| \alpha_{A}\right\rangle \\
\vdots \\
\left\langle\alpha_{M_{l}}^{\prime}\left|\dot{\mathbf{A}}_{j}\right| \alpha_{A}\right\rangle
\end{array}\right) .
$$

Taking the inner or scalar product of $\left(\left\langle\alpha_{A}\left|\mathbf{A}_{i}\right| \alpha_{M_{1}}^{\prime} \ldots<\left\langle\alpha_{A}\left|\mathbf{A}_{i}\right| \alpha_{M_{l}}^{\prime}\right\rangle\right)\right.$ and its complex conjugate, and using (16) and (17), one obtains

$$
\begin{gathered}
\left.\left(\left\langle\alpha_{A}\left|\mathbf{A}_{i}\right| \alpha_{M_{1}}^{\prime}\right\rangle \ldots<\alpha_{A}\left|\mathbf{A}_{i}\right| \alpha_{M_{l}}^{\prime}\right\rangle\right)\left(\begin{array}{c}
<\alpha_{M_{1}}^{\prime}\left|\mathbf{A}_{i}\right| \alpha_{A}> \\
\vdots \\
\left.<\alpha_{M_{l}}^{\prime}\left|\dot{\mathbf{A}}_{i}\right| \alpha_{A}\right\rangle
\end{array}\right) \\
\left.=\left(\left\langle\alpha_{A}\left|\mathbf{A}_{j}\right| \alpha_{M_{1}}^{\prime}\right\rangle \ldots<\alpha_{A}\left|\mathbf{A}_{j}\right| \mathbf{A} \alpha_{M_{l}}^{\prime}\right\rangle\right) \mathbf{T T}\left(\begin{array}{c}
<\alpha_{M_{1}}^{\prime}\left|\mathbf{A}_{j}\right| \alpha_{A}> \\
\vdots \\
\left.<\alpha_{M_{l}}^{\prime}\left|\dot{A}_{j}\right| \alpha_{A}\right\rangle
\end{array}\right)
\end{gathered}
$$

or

$$
\left.\sum_{l}\left\langle\alpha_{A}\left|\mathbf{A}_{i}\right| \alpha_{M_{l}}^{\prime}\right\rangle\left\langle\alpha_{M_{l}}^{\prime}\left|\mathbf{A}_{i}\right| \alpha_{A}\right\rangle=\sum_{l}<\alpha_{A}\left|\mathbf{A}_{j}\right| \alpha_{M_{l}}^{\prime}\right\rangle\left\langle\alpha_{M_{l}}^{\prime}\left|\mathbf{A}_{j}\right| \alpha_{A}\right|>
$$

Consequently, the sum of the square moduli of the one particle transition integrals involving $\mathbf{A}_{i}$ and $\mathbf{A}_{j}$ between an antisymmetric state and the degenerate states of mixed symmetry are equal.

In summary, the relationship between one particle transitions for systems of three or more electrons are ${ }^{4}$

$$
\begin{aligned}
& \left\langle\alpha_{A}\left|\mathbf{A}_{i}\right| \alpha_{A}^{\prime}\right\rangle=\left\langle\alpha_{A}\left|\mathbf{A}_{j}\right| \alpha_{A}^{\prime}\right\rangle \\
& \left\langle\alpha_{A}\left|\mathbf{A}_{i}\right| \alpha_{S}^{\prime}\right\rangle=\left\langle\alpha_{A}\left|\mathbf{A}_{j}\right| \alpha_{S}^{\prime}\right\rangle=0 \\
& \sum_{l}\left|\left\langle\alpha_{A}\left|\mathbf{A}_{i}\right| \alpha_{M l}^{\prime}\right\rangle\right|^{2}=\sum_{l}\left|\left\langle\alpha_{A}\left|\mathbf{A}_{j}\right| \alpha_{M_{l}}^{\prime}\right\rangle\right|^{2} .
\end{aligned}
$$

\section{Nature of Two Particle Correlations in Terms of One Particle Transitions}

It will be assumed in this discussion that any many-particle operator of interest can be expanded in products of one particle operators. Since the main features of the matrix sum expansion of the expectation value of a product of one particle operators is illustrated by the expansion of a product of two one particle operators, the following discussion will center its attention on the two particle operator $\mathbf{A}_{i} \cdot \mathbf{A}_{j}$.

For a two electron system, the discussion of the previous section indicates that

$$
\begin{aligned}
\left\langle\alpha\left|\mathbf{A}_{1} \cdot \mathbf{A}_{2}\right| \alpha\right\rangle & =\sum_{\alpha_{A^{\prime}}}\left\langle\alpha_{A}\left|\mathbf{A}_{1}\right| \alpha_{A}^{\prime}\right\rangle\left\langle\alpha_{A}^{\prime}\left|\mathbf{A}_{2}\right| \alpha_{A}\right\rangle \\
& +\sum_{\alpha S^{\prime}}\left\langle\alpha_{A}\left|\mathbf{A}_{1}\right| \alpha_{S}^{\prime}\right\rangle\left\langle\alpha_{S}^{\prime}\left|\mathbf{A}_{2}\right| \alpha_{A}\right\rangle \\
& =\sum_{\alpha_{A^{\prime}}}\left|\left\langle\alpha_{A}\left|\mathbf{A}_{1}\right| \alpha_{A}^{\prime}\right\rangle\right|^{2}-\sum_{\alpha_{S^{\prime}}}\left|\left\langle\alpha_{A}\left|\mathbf{A}_{1}\right| \alpha_{S}^{\prime}\right\rangle\right|^{2}
\end{aligned}
$$

where the sum $\alpha_{A}^{\prime}$ is over the antisymmetric states and the sum $\alpha_{S}^{\prime}$ is over the symmetric states. It is apparent that for the expectation value of $\mathbf{A}_{1} \cdot \mathbf{A}_{2}$ to be zero, the sums of the square moduli of the transitions involving $\mathbf{A}_{1}$ over all antisymmetric states must equal the sum over all symmetric states. This is not the case in general, unless the electrons are isolated and do not interact. Moreover, it is apparent that the sign of the expectation value is dependent upon which of the two sums is larger in magnitude.

${ }^{4}$ For a system of Bosons, the above rules would be $\left\langle\alpha_{S}\left|\mathbf{A}_{i}\right| \alpha_{S}^{\prime}\right\rangle=\left\langle\alpha_{S}\left|\mathbf{A}_{j}\right| \alpha_{S}^{\prime}\right\rangle,\left\langle\alpha_{S}\left|\mathbf{A}_{i}\right| \alpha_{A}^{\prime}\right\rangle=\left\langle\alpha_{S}\left|\mathbf{A}_{j}\right| \alpha_{A}^{\prime}\right\rangle=0$, and $\Sigma \mid\left\langle\alpha_{S}\left|\mathbf{A}_{i}\right| \alpha_{M_{l}}^{\prime}>\left.\right|^{2}=\Sigma\left|<\alpha_{S}\right| \mathbf{A}_{i}\left|\alpha_{M l}^{\prime}>\right|{ }^{2}\right.$. 
In the case of three or more electrons, one considers the operator $\sum_{i \neq j} \mathbf{A}_{i} \cdot \mathbf{A}_{j}$. The expectation value of this operator is given by

$$
\begin{aligned}
\sum_{i \neq j}\left\langle\alpha\left|\mathbf{A}_{i} \cdot \mathbf{A}_{j}\right| \alpha\right\rangle & =\sum_{i \neq j} \sum_{\alpha_{A^{\prime}}^{\prime}}\left\langle\alpha_{A}\left|\mathbf{A}_{i}\right| \alpha_{A}^{\prime}\right\rangle\left\langle\alpha_{A}^{\prime}\left|\mathbf{A}_{j}\right| \alpha_{A}\right\rangle \\
& +\sum_{i \neq j} \sum_{\alpha_{M^{\prime}}}\left\langle\alpha_{A}\left|\mathbf{A}_{i}\right| \alpha_{M}^{\prime}\right\rangle\left\langle\alpha_{M}^{\prime}\left|\mathbf{A}_{j}\right| \alpha_{A}\right\rangle .
\end{aligned}
$$

If one notes that $\left\langle\alpha_{M}^{\prime}\left|\sum_{j=1}^{N} \mathbf{A}_{j}\right| \alpha_{A}\right\rangle=0$, then $\left\langle\alpha_{M}^{\prime}\left|\sum_{j \neq i} \mathbf{A}_{j}\right| \alpha_{A}\right\rangle=-\left\langle\alpha_{M}^{\prime}\left|\mathbf{A}_{i}\right| \alpha_{A}\right\rangle$. Consequently, (20) may be written:

$$
\sum_{i \neq j}\left\langle\alpha\left|\mathbf{A}_{i} \cdot \mathbf{A}_{j}\right| \alpha\right\rangle=N(N-1) \sum_{\alpha_{A}^{\prime}}\left|\left\langle\alpha_{A}\left|\mathbf{A}_{1}\right| \alpha_{A}^{\prime}\right\rangle\right|^{2}-N \sum_{\alpha_{M^{\prime}}}\left|\left\langle\alpha_{A}\left|\mathbf{A}_{1}\right| \alpha_{M}^{\prime}\right\rangle\right|^{2}
$$
It may be seen that the magnitude and sign of the expectation value of $\sum_{i \neq j} \mathbf{A}_{i} \cdot \mathbf{A}_{j}$ is dependent
upon the relative magnitudes of the sums

$$
\sum_{\alpha_{M^{\prime}}}\left|\left\langle\alpha_{A}\left|\mathbf{A}_{1}\right| \alpha_{M}^{\prime}\right\rangle\right|^{2} \text { and }(N-1) \sum_{\alpha_{A^{\prime}}}\left|\left\langle\alpha_{A}\left|\mathbf{A}_{1}\right| \alpha_{A}^{\prime}\right\rangle\right|^{2} .
$$

Equations (19) and (21) may be combined to read for the general case

$$
\sum_{i \neq j}\left\langle\alpha\left|\mathbf{A}_{i} \cdot \mathbf{A}_{j}\right| \alpha\right\rangle=N(N-1) \sum_{\alpha_{A^{\prime}}^{\prime}}\left|\left\langle\alpha\left|\mathbf{A}_{1}\right| \alpha_{A}^{\prime}\right\rangle\right|^{2}-N \sum_{\alpha_{N^{\prime}}}\left|\left\langle\alpha_{A}\left|\mathbf{A}_{1}\right| \alpha_{N}^{\prime}\right\rangle\right|^{2}
$$

where the sum $\alpha_{N}^{\prime}$ is over all the non-antisymmetric states.

We are now in a position to discuss the physical nature of the two particle correlations. From a conceptional point of view, the expectation value $\left\langle\alpha\left|\mathbf{A}_{i} \cdot \mathbf{A}_{j}\right| \alpha\right\rangle$ may be given a geometrical meaning. If $\mathbf{A}_{i}$ and $\mathbf{A}_{j}$ are represented as vector quantities, then the above expectation value may be represented as their scalar product $\left|\mathbf{A}_{i}\right|\left|\mathbf{A}_{j}\right| \cos \gamma$, where $\left|\mathbf{A}_{i}\right|$ and $\left|\mathbf{A}_{j}\right|$ are the average magnitudes of the vectors given by $\left|\mathbf{A}_{i}\right|^{2}=\left\langle\alpha\left|\mathbf{A}_{i x}^{2}+\mathbf{A}_{i y}^{2}+\mathbf{A}_{i z}^{2}\right| \alpha\right\rangle$ and $\gamma$ is the average angle between them. Since the average values of the magnitudes of $\mathbf{A}_{i}$ and $\mathbf{A}_{j}$ are equal because of the indistinguishability principle, the scalar product reduces to $\left|\mathbf{A}_{1}\right|^{2} \cos \gamma$.

The above discussion may now be applied to the derivation of an important approximate relation between the ratios $\left\langle\alpha\left|\mathbf{A}_{i}^{2}\right| \alpha\right\rangle /\left\langle\alpha\left|\mathbf{B}_{i}^{2}\right| \alpha\right\rangle$ and $\left\langle\alpha\left|\mathbf{A}_{i} \cdot \mathbf{A}_{j}\right| \alpha\right\rangle \mid\left\langle\alpha\left|\mathbf{B}_{i} \cdot \mathbf{B}_{j}\right| \alpha\right\rangle$, where the $\mathbf{A}_{i}$ and $\mathbf{B}_{i}$ refer to two different operators. Beginning with the assumption $\left\langle\alpha\left|\mathbf{A}_{i}^{2}\right| \alpha\right\rangle \gg\left\langle\alpha\left|\mathbf{A}_{i} \cdot \mathbf{A}_{j}\right| \alpha\right\rangle$, for any $\mathbf{A}_{i}$, we make the approximation

$$
\frac{\left\langle\alpha\left|\mathbf{A}_{1}^{2}\right| \alpha\right\rangle}{\left\langle\alpha\left|\mathbf{B}_{1}^{2}\right| \alpha\right\rangle} \approx \frac{\left\langle\alpha\left|\left(\sum_{i} \mathbf{A}_{i}\right)^{2}\right| \alpha\right\rangle}{\left\langle\alpha\left|\left(\sum_{i} \mathbf{B}_{i}\right)^{2}\right| \alpha\right\rangle} .
$$

Now

where

$$
\frac{\left\langle\alpha\left|\mathbf{A}_{1}^{2}\right| \alpha\right\rangle}{\left\langle\alpha\left|\mathbf{B}_{1}^{2}\right| \alpha\right\rangle}=\frac{Q+T}{S+P}
$$

$$
\begin{aligned}
& Q=\sum_{\alpha_{N}^{\prime}}\left|\left\langle\alpha\left|\mathbf{A}_{1}\right| \alpha_{A}^{\prime}\right\rangle\right|^{2} \\
& T=\sum_{\alpha_{N}^{\prime}}\left|\left\langle\alpha\left|\mathbf{A}_{1}\right| \alpha_{N}^{\prime}\right\rangle\right|^{2} \\
& S=\sum_{\alpha_{A^{\prime}}}\left|\left\langle\alpha\left|\mathbf{B}_{1}\right| \alpha_{A}^{\prime}\right\rangle\right|^{2} \\
& P=\sum_{\alpha_{N^{\prime}}}\left|\left\langle\alpha\left|\mathbf{B}_{1}\right| \alpha_{N}^{\prime}\right\rangle\right|^{2}
\end{aligned}
$$

On the other hand, it is evident that $\left(\sum_{i} \mathbf{A}_{i}\right)^{2}$ and $\left(\sum_{i} \mathbf{B}_{i}\right)^{2}$ are symmetric operators so that in the 
expansion of their expectation values, only transitions to antisymmetric states need be considered. Consequently:

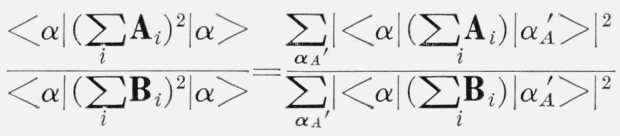

$$
\begin{aligned}
& =\frac{\sum_{\alpha_{A^{\prime}}}\left|\left\langle\alpha\left|N \mathbf{A}_{1}\right| \alpha_{A}^{\prime}\right\rangle\right|^{2}}{\sum_{\alpha A^{\prime}}^{\prime}\left|\left\langle\alpha\left|N \mathbf{B}_{1}\right| \alpha_{A}^{\prime}\right\rangle\right|^{2}}=\frac{Q}{S} .
\end{aligned}
$$

We now consider the ratio of the two particle correlation terms. The use of eq (22) yields

$$
\frac{\sum_{i \neq j}\left\langle\alpha\left|\mathbf{A}_{i} \cdot \mathbf{A}_{j}\right| \alpha\right\rangle}{\sum_{i \neq j}\left\langle\alpha\left|\mathbf{B}_{i} \cdot \mathbf{B}_{j}\right| \alpha\right\rangle}=\frac{N(N-1) Q-N T}{N(N-1) S-N P}
$$

which upon application of the indistinguishability principle becomes

$$
\frac{\left\langle\alpha\left|\mathbf{A}_{1} \cdot \mathbf{A}_{2}\right| \alpha\right\rangle}{\left\langle\alpha\left|\mathbf{B}_{1} \cdot \mathbf{B}_{2}\right| \alpha\right\rangle}=\frac{(N-1) Q-T}{(N-1) R-P} .
$$

It is apparent from (23), (24), and (25) that in this approximation:

from which we obtain

$$
\frac{Q+T}{S+P} \approx \frac{Q}{S}
$$

$$
T \approx \frac{Q P}{S}
$$

Substitution of this result in eq (27) yields the result:

$$
\frac{\left\langle\alpha\left|\mathbf{A}_{1} \cdot \mathbf{A}_{2}\right| \alpha\right\rangle}{\left\langle\alpha\left|\mathbf{B}_{1} \cdot \mathbf{B}_{2}\right| \alpha\right\rangle} \approx \frac{(N-1) \mathrm{Q}-\frac{Q P}{S}}{(N-1) S-P}=\frac{Q}{S}
$$

from which we conclude

$$
\frac{\left\langle\alpha\left|\mathbf{A}_{1}^{2}\right| \alpha\right\rangle}{\left\langle\alpha\left|\mathbf{B}_{1}^{2}\right| \alpha\right\rangle} \approx \frac{\left\langle\alpha\left|\mathbf{A}_{1} \cdot \mathbf{A}_{2}\right| \alpha\right\rangle}{\left\langle\alpha\left|\mathbf{B}_{1} \cdot \mathbf{B}_{2}\right| \alpha\right\rangle} \text {. }
$$

An equivalent relation is

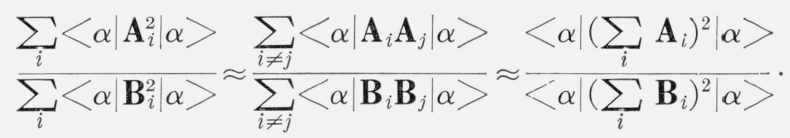

In the limit when the ratios $\left\langle\alpha\left|\mathbf{A}_{1} \cdot \mathbf{A}_{2}\right| \alpha\right\rangle /\left\langle\alpha\left|\mathbf{A}_{1}^{2}\right| \alpha\right\rangle$ and $\left\langle\alpha\left|\mathbf{B}_{1} \cdot \mathbf{B}_{2}\right| \alpha\right\rangle \mid\left\langle\alpha\left|\mathbf{B}_{1}^{2}\right| \alpha\right\rangle$ both approach zero, the above approximate relations (30) and (31) become exact. One may conclude from equation (30) that in this limit, the correlation angles involving the two operators under consideration are equal. These relations are important in that they equate the ratio of two correlation terms to ratios of quantities which are observables, at least in principle.

Because of the importance of these relations, a few remarks regarding their applicability and range of validity are pertinent. The derivation of eqs (30) and (31) establishes the equality of the correlation angles associated with all two particle operators of the type $\mathbf{A}_{1} \cdot \mathbf{A}_{2}$ when correlation effects are very small. Such a situation occurs in systems consisting of very weakly interacting particles. This result is not trivial since a weak interaction only necessarily implies that the quantities $\cos \gamma$ for different operators are small, and not that there is necessarily any relationship between them in the limit of vanishing interaction. 
It is now of value to analyze the applicability of these relations to a system where the interparticle interactions are not necessarily small. One might expect, in this case, that a more realistic assumption upon which to base the above derivation is that $\left\langle\alpha\left|\mathbf{A}_{i}^{2}\right| \alpha\right\rangle$ must be very large compared to $(N-1)\left\langle\alpha\left|\mathbf{A}_{i} \cdot \mathbf{A}_{j}\right| \alpha\right\rangle$ for an $N$ particle system, since there are $N$ terms of the first type and $N(N-1)$ terms of the second type. The factor $(N-1)$ would introduce an order of magnitude increase in the size of the small quantity and would, of course, place some doubt upon the validity of the initial assumption.

For most real systems, however, this consideration does not present a serious problem, primarily because the particles of a system generally arrange themselves in groups which are not strongly correlated. An example of this is the electronic shells of atoms. Consequently, the correlation terms do not generally become more important for systems with large numbers of particles than for systems containing few particles.

A check on this conclusion is possible in the case when the operators $\mathbf{A}_{i}$ involved are the radius vectors $\mathbf{r}_{i}$ of the electrons in an atom or molecule. Kirkwood and Vinti, ${ }^{5}$ using different techniques, independently derived an approximate expression relating the electric and diamagnetic susceptibilities of closed shell atoms and molecules. However, Kirkwood's expression neglects the contribution due to the correlation term $\sum_{i \neq j}\left\langle\alpha\left|\mathbf{r}_{i} \cdot \mathbf{r}_{j}\right| \alpha\right\rangle$. Since Kirkwood's calculated diamagnetic susceptibilities for several rare gases and diatomic hydrogen do not differ from the observed values by more than a few percent, it appears safe to conclude that $\sum_{i}\left\langle\alpha\left|\mathbf{r}_{i}^{2}\right| \alpha\right\rangle$ is generally at least an order of magnitude greater than $\sum_{i \neq j}\left\langle\alpha\left|\mathbf{r}_{i} \cdot \mathbf{r}_{j}\right| \alpha\right\rangle$.

The question still remains as to whether this ratio is great enough to insure the validity of eqs (30) and (31) when applied to most systems. In order to arrive at a definitive answer to this question, one must first recognize that the fundamental assumption (23) involved in the derivation of these equations is valid not only in the limit of vanishing correlation, but also in the case when the correlation angles involved are equal or do not differ greatly in magnitude. Since we have already established that the correlation angles are equal for vanishingly small correlation, it is reasonable to conclude that the correlation angles are very nearly (if not actually) equal in the case when $\sum_{i \neq j}\left\langle\alpha\left|\mathbf{A}_{i} \cdot \mathbf{A}_{j}\right| \alpha\right\rangle$ is an order of magnitude smaller than $\sum_{i}\left\langle\alpha\left|\mathbf{A}_{i}^{2}\right| \alpha\right\rangle$. Consequently, we may conclude that in most cases, the approximation (23) is a good one, and equations (30) and (31) are valid. This conclusion is of great practical value, since these equations enable one to calculate the value of any correlation term, given the appropriate observables, once any one such term is evaluated either indirectly or by direct theoretical calculation. An example of such a calculation will be discussed in the next section.

\section{Derivation and Application of a Sum Rule for One Particle Transitions}

Sum rules, in general, are useful in providing information about a system without a detailed knowledge of its wave functions or energy levels. In the case of sum rules which involve one particle transitions, information can be obtained regarding the relative magnitudes of one particle transitions as well as the relative energy separations of the various quantum states (observable or unobservable) of the system. In some cases, information can be obtained regarding the expectation value of many-particle operators.

One useful sum rule of this type follows from the $N$ electron nonrelativistic Hamiltonian

$$
\mathbf{H}=(1 / 2 m) \sum_{i} \mathbf{p}_{i}^{2}+V\left(\mathbf{r}_{1} \mathbf{r}_{2} \ldots \mathbf{r}_{N}\right)
$$

and the Heisenberg commutation relations

$$
\mathbf{r}_{i} \mathbf{p}_{j}-\mathbf{p}_{j} \mathbf{r}_{i}=3 i \hbar \delta_{i 1}
$$

${ }_{5}^{5}$ J. G. Kirkwood, Phys. Zeits. 33, 57 (1932); J. P. Vinti, Phys. Rev. 41, 813 (1932). 


$$
\begin{gathered}
\mathbf{r}_{i} \mathbf{r}_{j}-\mathbf{r}_{j} \mathbf{r}_{i}=0 \\
\mathbf{p}_{i} \mathbf{p}_{j}-\mathbf{p}_{j} \mathbf{p}_{i}=0
\end{gathered}
$$

From (32), (33), (34), and (35), it follows that

$$
\mathbf{p}_{i}=-(i m / \hbar)\left(\mathbf{r}_{i} \mathbf{H}-\mathbf{H r}_{i}\right)
$$

so that the matrix components of $\mathbf{p}_{i}$ in terms of those of $\mathbf{r}_{i}$ are

$$
\left\langle\alpha\left|\mathbf{p}_{i}\right| \alpha^{\prime}\right\rangle=-\frac{i m}{\hbar} E_{\alpha^{\prime} \alpha}\left\langle\alpha\left|\mathbf{r}_{i}\right| \alpha^{\prime}\right\rangle
$$

where $E_{\alpha^{\prime} \alpha}=E_{\alpha^{\prime}}-E_{\alpha}$. Since $\left\langle\alpha\left|\mathbf{r}_{i} \mathbf{p}_{i}-\mathbf{p}_{i} \mathbf{r}_{i}\right| \alpha\right\rangle=3 i \hbar$, then

$$
\sum_{\alpha^{\prime}} E_{\alpha^{\prime} \alpha}\left|\left\langle\alpha\left|\mathbf{r}_{i}\right| \alpha^{\prime}\right\rangle\right|^{2}=3 \hbar^{2} / 2 m
$$

Since the sum is the same for all the $N$ particles, we conclude that

$$
\sum_{i=1} \sum_{\alpha^{\prime}} E_{\alpha^{\prime} \alpha} \mid\left\langle\alpha\left|\mathbf{r}_{i}\right| \alpha^{\prime}>\left.\right|^{2}=3 \hbar^{2} N / 2 m\right.
$$

It is apparent that these sum rules confirm the result (18) for the special case when $\mathbf{A}_{i}=\mathbf{r}_{i}$, since they can only be correct, in general, if the sum of the square moduli of the transitions involving: $\mathbf{r}_{i}$ and $\mathbf{r}_{j}$ to degenerate states are equal.

The sum rules (38) and (39) resemble the well-known Thomas-Kuhn-Reiche ${ }^{6}$ sum rule for real transitions

$$
\left.\sum_{\alpha^{\prime}} E_{\alpha^{\prime} \alpha}|<| \alpha|\mathbf{R}| \alpha^{\prime}\right\rangle\left.\right|^{2}=3 \hbar^{2} N / 2 m
$$

where $\mathbf{R}=\sum_{i=1}^{N} \mathbf{r}_{i}$. This sum rule may be obtained by summing eq (37) over all the electrons and noting that $\langle\alpha|\mathbf{R P}-\mathbf{P R}| \alpha\rangle=3 N i \hbar$, where $\mathbf{P}=\sum_{i=1}^{N} \mathbf{p}_{i}$.

Comparison of (39) and (40) confirms the fact that transitions to unobservable quantum states are decisive in validating sums which involve one particle transitions. If the sum (39) only involved transitions to antisymmetric states, it would differ from the sum (40) by a factor of $N$. From equation (40), it is evident that

$$
\sum_{i=1}^{N} \sum_{\alpha_{A}^{\prime}} E_{\alpha_{A}^{\prime} \alpha_{A}}\left|\left\langle\alpha_{A}\left|\mathbf{r}_{i}\right| \alpha_{A}^{\prime}\right\rangle\right|^{2}=3 \hbar^{2} / 2 m
$$

where the sum in (41) is over one particle transitions to antisymmetric states only. As a result, one may conclude that

$$
\sum_{i=1}^{2} \sum_{\alpha_{S}^{\prime}} E_{\alpha_{S}^{\prime} \alpha_{A}}\left|\left\langle\alpha_{A}\left|\mathbf{r}_{i}\right| \alpha_{S}^{\prime}\right\rangle\right|^{2}=3 \hbar^{2} / 2 m
$$

for a two electron system, where the sum (42) is over transitions to symmetric states only, while

$$
\left.\sum_{i=1}^{N} \sum_{\alpha_{M}^{\prime}} E_{\alpha_{M} \alpha_{A}}\left|<\alpha_{A}\right| \mathbf{r}_{i}\left|a_{M}^{\prime}\right\rangle\right|^{2}=(N-1) 3 \hbar^{2} / 2 m
$$

for a three or more electron system, where the sum (43) is over transitions to mixed states only. If one subtracts (39) from (40) one finds that

$$
\sum_{i \neq j} \sum_{\alpha^{\prime}} E_{\alpha^{\prime} \alpha}\left\langle\alpha\left|\mathbf{r}_{i}\right| \alpha^{\prime}\right\rangle\left\langle\alpha^{\prime}\left|\mathbf{r}_{j}\right| \alpha\right\rangle=0 .
$$

\footnotetext{
${ }^{6}$ W. Thomas, Naturwissenschaften 13, 627 (1925); W. Kuhn, Z. Physik 33, 408 (1925); F. Reiche and W. Thomas, Z. Physik 34, 510 (1925).
} 
This result would appear to be paradoxical if one sums (44) by means of an average energy $\bar{E}$ such that (44) becomes

$$
\bar{E} \sum_{\alpha^{\prime}} \sum_{i \neq j}^{\prime}\left\langle\alpha\left|\mathbf{r}_{i}\right| \alpha^{\prime}\right\rangle\left\langle\alpha^{\prime}\left|\mathbf{r}_{j}\right| \alpha\right\rangle=\bar{E} \sum_{i \neq j}^{\prime}\left\langle\alpha\left|\mathbf{r}_{i} \cdot \mathbf{r}_{j}\right| \alpha\right\rangle .
$$

Since $\left\langle\alpha\left|\sum_{i \neq j} \mathbf{r}_{i} \cdot \mathbf{r}_{j}\right| \alpha\right\rangle$ is not equal to zero in general, this result indicates that $\bar{E}$ always vanishes. Although the invariably null value of the average energy difference seems to be a strange result, the correctness of the sum (44) can be directly demonstrated from the commutation relations. By application of (37), one may see that

$$
\begin{aligned}
\sum_{i \neq j} \sum_{\alpha^{\prime}} E_{\alpha^{\prime} \alpha}\left\langle\alpha\left|\mathbf{r}_{i}\right| \alpha^{\prime}\right\rangle\left\langle\alpha^{\prime}\left|\mathbf{r}_{i}\right| \alpha\right\rangle=-\frac{\hbar}{2 i m} \sum_{i \neq} \sum_{\alpha^{\prime}}\left\langle\alpha\left|\mathbf{r}_{i}\right| \alpha^{\prime}\right\rangle\left\langle\alpha^{\prime}\left|\mathbf{p}_{i}\right| \alpha\right\rangle & \\
& +\frac{\hbar}{2 i m} \sum_{i \neq j} \sum_{\alpha^{\prime}}\left\langle\alpha\left|\mathbf{p}_{j}\right| \alpha^{\prime}\right\rangle\left\langle\alpha^{\prime}\left|\mathbf{r}_{i}\right| \alpha\right\rangle
\end{aligned}
$$

where in the second sum, the dummy indices $i$ and $j$ have been interchanged. The right hand side of (45) is simply, from the sum rule for matrix multiplication $-\frac{\hbar}{2 i m} \sum_{i \neq i}\left\langle\alpha\left|\mathbf{r}_{i} \cdot \mathbf{p}_{j}-\mathbf{p}_{j} \cdot \mathbf{r}_{i}\right| \alpha\right\rangle$ which is zero as a result of the commutation relation (33). ${ }^{7}$

The origin of the vanishing average energy difference can be demonstrated if one rewrites the sum (44) in the following manner:

$$
\sum_{i \neq j}\left(\sum_{\alpha_{A}^{\prime}} E_{\alpha_{A}^{\prime} \alpha_{A}}^{\prime}\left\langle\alpha\left|\mathbf{r}_{i}\right| \alpha_{A}^{\prime}\right\rangle\left\langle\alpha_{A}^{\prime}\left|\mathbf{r}_{j}\right| \alpha_{A}\right\rangle+\sum_{\alpha^{\prime}} E_{\alpha_{N}^{\prime} \alpha_{A}}\left\langle\alpha_{A}\left|\mathbf{r}_{i}\right| \alpha_{N}^{\prime}\right\rangle\left\langle\alpha_{N}^{\prime}\left|\mathbf{r}_{j}\right| \alpha_{A}\right\rangle\right)
$$

where the sum $\alpha_{N}^{\prime}$ is over all the states which are not antisymmetric, i.e., the symmetric states for $N=2$, and the mixed states for $N \geq 3$. The sum (46) can be written in the form

$$
\begin{aligned}
\sum\left((N-1) \bar{E}_{A} \sum_{\alpha_{A}^{\prime}}\left|\left\langle\alpha_{A}\left|\mathbf{r}_{i}\right| \alpha_{A}^{\prime}\right\rangle\right|^{2}-\bar{E}_{N} \sum_{\alpha_{N}^{\prime}}\left|\left\langle\alpha_{A}\left|\mathbf{r}_{i}\right| \alpha_{N}^{\prime}\right\rangle\right|^{2}\right) \\
=N(N-1) \bar{E}_{A} \sum_{\alpha_{A}^{\prime}}\left|\left\langle\alpha_{A}\left|\mathbf{r}_{1}\right| \alpha_{A}^{\prime}\right\rangle\right|^{2}-N \bar{E}_{N} \sum_{\alpha_{N}^{\prime}}\left|\left\langle\alpha_{A}\left|\mathbf{r}_{1}\right| \alpha_{N}^{\prime}\right\rangle\right|^{2}
\end{aligned}
$$

where $\bar{E}_{A}$ and $\bar{E}_{N}$ are the average energy differences between the ground state and the antisymmetric states, and the ground state and the non-antisymmetric states, respectively.

For the special case, when $\left\langle\alpha\left|\mathbf{r}_{i} \cdot \mathbf{r}_{j}\right| \alpha\right\rangle=0$, i.e., when the electrons are uncorrelated and the system has no electric dipole moment, then $N(N-1) \sum\left|\underset{\alpha_{A}^{\prime}}{<\alpha_{A}}\right| \mathbf{r}_{1}\left|\alpha_{A}^{\prime}\right|^{2}-\left.N \sum\left|\underset{\alpha_{N}^{\prime}}{<\alpha_{A}}\right| \mathbf{r}_{1}\left|\alpha_{N}^{\prime}\right\rangle\right|^{2}=0$ and $\bar{E}_{A}$ and $\bar{E}_{N}$ must be equal. In all other cases, however, these two quantities are not equal, but, instead, their values are adjusted so that $N(N-1) \bar{E}_{A} \sum_{\alpha_{A}}\left|\left\langle\alpha_{A}\left|\mathbf{r}_{1}\right| \alpha_{A}^{\prime}\right\rangle\right|^{2}$ always equals $N \bar{E}_{N} \sum_{\alpha_{N}^{\prime}}\left|\left\langle\alpha_{A}\left|\mathbf{r}_{1}\right| \alpha_{A}^{\prime}\right\rangle\right|^{2}$. As seen in this light, the vanishing total average energy difference results from the fact that it is a composite of average energy differences involving virtual one particle transitions to states of different symmetry. This special relationship that exists in the nonrelativistic approximation between these weighted mean energy differences and the transition integrals of the type $\left\langle\alpha_{A}\left|\mathbf{r}_{1}\right| \alpha_{A}^{\prime}\right\rangle$ and $\left\langle\alpha_{A}\left|\mathbf{r}_{1}\right| \alpha_{N}^{\prime}\right\rangle$ is a restriction on all systems, regardless of their detailed properties, which is imposed by the nature of the Schrodinger representation, which in turn follows from the commutation relations.

The sum rules (38) and (39) have immediate application in a demonstration of an approximate relationship between the diamagnetic susceptibility and the total energy of a system in the nonrelativistic approximation. If one assumes that the ground state of the system does

7 The author is indebted to Dr. J. P. Vinti for clarifying this point. 
not have an electric dipole moment, i.e., that

$$
\langle\alpha|X| \alpha\rangle=N\left\langle\alpha\left|x_{1}\right| \alpha\right\rangle=0=\left\langle\alpha\left|y_{i}\right| \alpha\right\rangle=\left\langle\alpha\left|z_{i}\right| \alpha\right\rangle
$$

then (38) may be written

$$
\bar{E}_{r_{i}} \sum_{\alpha}\left|\left\langle\alpha\left|\mathbf{r}_{i}\right| \alpha^{\prime}\right\rangle\right|^{2}=\bar{E}_{r_{i}}\left\langle\alpha\left|\mathbf{r}_{i}^{2}\right| \alpha\right\rangle=3 \hbar^{2} / 2 m .
$$

On the other hand, (48) may be rewritten in the following fashion by making use of (37):

$$
\sum_{\alpha^{\prime}} E_{\alpha^{\prime} \alpha}\left|\left\langle\alpha\left|\mathbf{r}_{i}\right| \alpha^{\prime}\right\rangle\right|^{2}=3 \hbar^{2} / 2 m=\left(\frac{\hbar}{m}\right)^{2} \sum_{\alpha^{\prime}} \frac{\left|\left\langle\alpha\left|\mathbf{p}_{i}\right| \alpha^{\prime}\right\rangle\right|^{2}}{E_{\alpha^{\prime} \alpha}}
$$

Equation (49) may now be written as

$$
1 / \bar{E}_{p_{i}}\left(\frac{\hbar}{m}\right)^{2} \sum_{\alpha^{\prime}}\left|\left\langle\alpha\left|\mathbf{p}_{i}\right| \alpha^{\prime}\right\rangle\right|^{2}=1 / \bar{E}_{p_{i}}\left(\frac{\hbar}{m}\right)^{2}\left\langle\alpha\left|\mathbf{p}_{i}^{2}\right| \alpha\right\rangle=3 \hbar^{2} / 2 m
$$

since $\sum_{i}\left\langle\alpha\left|\mathbf{p}_{i}\right| \alpha\right\rangle$ vanishes for any bound state. Comparison of (48) and (50) reveals that if the approximation $\bar{E}_{p_{i}}=\bar{E}_{r_{i}}$ is made, then the simple relationship

$$
\left\langle\alpha\left|\mathbf{r}_{i}^{2}\right| \alpha\right\rangle\left\langle\alpha\left|\mathbf{p}_{i}^{2}\right| \alpha\right\rangle=(3 \hbar / 2)^{2}
$$

results. One may note the similarity between (51) and the well known expression for the uncertainty principle.

When eqs (48) and (50) are summed over all the electrons, the following equations are obtained:

$$
\begin{aligned}
& \bar{E}_{r_{i}} \sum_{i=1}^{N}\left\langle\alpha\left|\mathbf{r}_{i}^{2}\right| \alpha\right\rangle=3 N \hbar^{2} / 2 m \\
& 1 / \bar{E}_{p_{i}} \sum_{i=1}^{N}\left\langle\alpha\left|\mathbf{p}_{i}^{2}\right| \alpha\right\rangle=3 N m / 2 .
\end{aligned}
$$

Using the expression for the diamagnetic susceptibility ${ }^{8}$

eq (42) becomes:

$$
\chi_{\mathrm{dia}}=-\frac{e^{2}}{6 m c^{2}} \sum_{i=1}^{\Lambda}\left\langle\alpha\left|\mathbf{r}_{i}^{2}\right| \alpha\right\rangle,
$$

$$
\chi_{\mathrm{dia}}=-\frac{N \hbar^{2} e^{2}}{4 m^{2} c^{2}} \frac{1}{\bar{E}_{r_{i}}} .
$$

Since the nonrelativistic Hamiltonian (32) contains only Coulombic interactions, by the virial theorem, the total energy of the system $W_{0}$ is equal to the negative of its kinetic energy $1 / 2 m \sum_{=1}^{N}\left\langle\alpha\left|\mathbf{p}_{i}^{2}\right| \alpha\right\rangle$. Making use of this fact, (53) becomes

$$
W_{0}=-\frac{3 N}{4} \bar{E}_{p_{i}}
$$

Combining (55) and (56), and again making use of the approxımation $\bar{E}_{p_{i}}=\bar{E}_{r_{i^{\prime}}}$ one obtains the simple relation between $\chi_{\mathrm{d} 1 \mathrm{a}}$ and $W_{0}$ :

where

$$
\chi_{\mathrm{d} 1 \mathrm{a}} W_{0}=K^{2}
$$

$$
K=\frac{\sqrt{3} N \hbar e}{4 m c} \text {. }
$$

\footnotetext{
8 J. H. Van Vleck, Theory of Electric and Magnetic Susceptibilities (Oxford Univ. Press, 1932).
} 
We are now in a position to examine the observable manifestations of the two particle correlations in the special case when the operators involved are the coordinates and moment of the particles. The expectation values of the operators $\sum_{i \neq j} \mathbf{r}_{i} \cdot \mathbf{r}_{j}$ and $\sum_{i \neq j} \mathbf{p}_{i} \cdot \mathbf{p}_{j}$ can be considered to be observable since they can be expressed as the difference between two quantities which are observable at least in principle. The first operator is equal to $\left(\sum_{i} \mathbf{r}_{i}\right)^{2}-\sum_{i} \mathbf{r}_{i}^{2}$; the expectation value of the first quantity is equal to the square of the extension of the system while, as discussed above, the expectation value of the second is proportional to the diamagnetic susceptibility of the system. Unfortunately, neither of these quantities is easy to determine with any degree of accuracy by experimental techniques except under special conditions. The diamagnetic susceptibility can be experimentally observed only if other magnetic effects, which would normally be dominant, vanish as is the case in closed shell atoms and molecules. The extension of the system is not easily observable, experimentally, although it is related to the electric polarizability (see footnote 5). The second operator is equal to $\left(\sum_{i} \mathbf{p}_{i}\right)^{2}-\sum_{i} \mathbf{p}_{i}^{2}$ which is again the difference between two observable quantities. A term proportional to the first operator, added to the Hamiltonian, exactly expresses the effect of the nuclear motion upon the energy of an atomic system. ${ }^{9}$ Consequently, the expectation value of this term gives, in first order perturbation theory, the shift in energy levels due to the nuclear motion. This can be observed directly in the relative shifts of the energy levels of different isotopes of a given atom. The expectation value of the second operator is, as discussed above, proportional to the total energy of the system.

We may now derive simplified relations for the ratio $\sum_{i \neq j}\left\langle\alpha\left|\mathbf{p}_{i} \cdot \mathbf{p}_{j}\right| \alpha\right\rangle / \sum_{i \neq j}\left\langle\alpha\left|\mathbf{r}_{i} \cdot \mathbf{r}_{j}\right| \alpha\right\rangle$. It is evident from the approximate relations (30) and (31) that

$$
\frac{\sum_{i \neq j}\left\langle\alpha\left|\mathbf{p}_{i} \cdot \mathbf{p}_{j}\right| \alpha\right\rangle}{\sum_{i \neq j}\left\langle\alpha\left|\mathbf{r}_{i} \cdot \mathbf{r}_{j}\right| \alpha\right\rangle} \approx \frac{\sum_{i}\left\langle\alpha\left|\mathbf{p}_{i}^{2}\right| \alpha\right\rangle}{\sum_{i}\left\langle\alpha\left|\mathbf{r}_{i}^{2}\right| \alpha\right\rangle}=\frac{e^{2}}{3 c^{2}} \frac{W_{0}}{\chi_{\mathrm{dia}}} .
$$

Although $W_{0}$ and $\chi_{\text {dia }}$ may be obtained experimentally or from the available wavefunction, a further simplification of this expression is useful. From eq (51), it may be seen that

$$
\frac{\sum_{i}\left\langle\alpha\left|\mathbf{p}_{i}^{2}\right| \alpha\right\rangle}{\sum\left\langle\alpha\left|\mathbf{r}_{i}^{2}\right| \alpha\right\rangle} \approx \frac{N^{2}(3 \hbar / 2)^{2}}{\left(\sum_{i}\left\langle\alpha\left|\mathbf{r}_{i}^{2}\right| \alpha\right\rangle\right)^{2}} .
$$

Consequently

$$
\sum_{i}\left\langle\alpha\left|\mathbf{p}_{j} \cdot \mathbf{p}_{j}\right| \alpha\right\rangle \approx(3 \hbar / 2)^{2} N^{2} \frac{\sum_{i \neq j}\left\langle\alpha\left|\mathbf{r}_{i} \cdot \mathbf{r}_{j}\right| \alpha\right\rangle}{\left(\sum_{i}\left\langle\alpha\left|\mathbf{r}_{i}^{2}\right| \alpha\right\rangle\right)^{2}} .
$$

Though approximate, this relation offers a considerable advantage in the calculation of the contribution of $\sum_{i \neq j}\left\langle\alpha\left|\mathbf{p}_{i} \cdot \mathbf{p}_{j}\right| \alpha\right\rangle$ to the isotope shift of atomic energy levels since it does not involve multiple differentiation of complicated wavefunctions.

Finally, it may be remarked that the sum rule ${ }^{10}$ for atomic transition probabilities

$$
\left(\frac{m}{h}\right)^{2} \sum_{\alpha^{\prime}} E_{\alpha^{\prime} \alpha}^{2}\left|\left\langle\alpha\left|\sum_{i} \mathbf{r}_{i}\right| \alpha\right\rangle\right|^{2}=2 m W_{0}+\sum_{i \neq j}\left\langle\alpha\left|\mathbf{p}_{i} \cdot \mathbf{p}_{j}\right| \alpha\right\rangle
$$

once again displays $\sum_{i \neq j}\left\langle\alpha\left|\mathbf{p}_{i} \cdot \mathbf{p}_{j}\right| \alpha\right\rangle$ as the difference between two observable quantities. The simplified formula (60) can be used to advantage in calculating its contribution to the sum.

\footnotetext{
9 D. S. Hughes and C. Eckart, Phys. Rev. 36, 694 (1930).
}

10 J. P. Vinti, Phys. Rev. 41, 432 (1932). 
For example, in the case of the helium atom, use of a wavefunction due to Hylleraas which gives the energy correct to one part in 3000 gives for $\sum_{i \neq j}\left\langle\alpha\left|\mathbf{r}_{i} \cdot \mathbf{r}_{j}\right| \alpha\right\rangle$ the value $-0.146 a_{0}^{2}$ and for $\sum_{i}\left\langle\alpha\left|\mathbf{r}_{i}^{2}\right| \alpha\right\rangle$, the value $+2.34 a_{0}^{2}$. From (60), it is easily found that $\sum_{i \neq j}\left\langle\alpha\left|\mathbf{p}_{i} \cdot \mathbf{p}_{j}\right| \alpha\right\rangle$ is $-.24 \hbar^{2} / a_{0}$.

This is about four percent of the value of $2 m W_{0}$ determined with the same wavefunction, so that this term amounts to a four percent correction in the value of the sum expressed in eq (61).

It may be further observed from the above discussion that $\sum_{i \neq j}\left\langle\alpha\left|\mathbf{p}_{i} \cdot \mathbf{p}_{j}\right| \alpha\right\rangle$ and $\sum_{i \neq j}\left\langle\alpha\left|\mathbf{r}_{i} \cdot \mathbf{r}_{j}\right| \alpha\right\rangle$ have the same sign. Since the electrons repel each other, one would expect the average angle between two radius vectors $\mathbf{r}_{i}$ and $\mathbf{r}_{j}$ to be greater than $\pi / 2$ in a spherically symmetric system so that $\sum_{i \neq j}\left\langle\alpha\left|\mathbf{r}_{i} \cdot \mathbf{r}_{j}\right| \alpha\right\rangle$ is negative as was determined above for He. Consequently, one may conclude, in general, that $\sum_{i \neq j}\left\langle\alpha\left|\mathbf{p}_{i} \cdot \mathbf{p}_{j}\right| \alpha\right\rangle$ is negative also under the same
conditions.

\section{Conclusion}

The main purpose of this article has been to demonstrate the nature of the many particle correlations in a system in terms of expansions involving unobservable one particle transitions between states of different symmetry. This conceptional approach has been explored and used to derive relations for some two particle operators which are of use in various types of calculations. However, the ultimate value of this approach lies in the application of sum rules which involve one particle transitions in an attempt to obtain information regarding the many particle correlations which has been largely intractable by other approximation schemes. This method of analysis will be explored in future publications.

The author is indebted to Dr. J. P. Vinti for several stimulating discussions regarding sum rules which involve virtual one particle transitions.

(Paper 69A2-338) 